\title{
LISTADO DE EVALUADORES/AS 2016
}

Aragón Monroy, Fernando - Instituto Nacional de Bellas Artes (INBA) - México

Ballarín Domingo, Pilar - Universidad de Granada - España

Bandini, Gianfranco - Università degli Studi Firenze - Italia

Bastos, Maria Helena - Pontíficia Universidade Católica do Rio Grande do Sul - Brasil

Berruezo Albeniz, Reyes - Universidad Pública de Navarra - España

Bressan, Edoardo - Università di Macerata - Italia

Bruno Jofré, Rosa - Queen's University - Canadá

Cagnolati, Antonella - Università di Foggia - Italia (2 evaluaciones)

Caroli, Dorena - Università di Macerata - Italia

Carreño Aguilar, Antonieta - Universitat de Barcelona - España

Carretero Rodríguez, Mario - Universidad Autónoma de Madrid - España

Caruso, Marcelo - Humboldt - Universität zu Berlin - Alemania

Carvalho, Marta Chagas de - Universidade de São Paulo - Brasil

Caspard, Pierre - Service d'histoire de l'éducation - Francia

Chartier, Anne Marie - Service d'Histoire de l'Éducation - Francia

Cid Fernández, Xosé Manuel - Universidade de Vigo - España

Colomer Rubio, Juan - Universitat de València - España

Comas Rubí, Francesca - Universitat de les Illes Balears - España

Cruz Orozco, José I. - Universitat de València - España

Díaz Cuyás, José - Universidad de La Laguna - España

Escolano Benito, Agustín - Universidad de Valladolid - España (2 evaluaciones)

Fernández Mellizo - Soto, Maria - Universidad Complutense de Madrid - España

Fernández Soria, Juan Manuel - Universitat de València - España (2 evaluaciones)

Fuentes Moreno, Concepción - Universitat de Barcelona - España

Gabriel Fernández, Narciso de - Universidade da Coruña - España

Ghizzoni, Carla - Università Cattolica del Sacro Cuore - Italia

Giomi, Fabio - EHESS - Ecole des hautes études en sciences sociales - Francia

Gómez García, Salvador - Universidad de Valladolid - España

Gouveia, Maria Cristina Soares de - Universidade Federal de Minas Gerais - Brasil 
Grana Gil, Isabel - Universidad de Málaga - España

Grosso, Luis - Universidade de Porto - Portugal

Guereña, Jean - Louis - Université François Rabelais, Tours - Francia

Holmen, Janne - Uppsala Universitet - Suecia

Hormigos Ruiz, Jaime - Universidad Rey Juan Carlos - España

Ibañez, Alex - Universidad del País Vasco - España

Inarejos Muñoz, Juan Antonio - Universidad de Extremadura - España

Iribarren Donadeu, Teresa - Universitat Oberta de Catalunya - España

Játiva Millares, María - Universitat d'Alacant - España

Jiménez Eguizábal, Alfredo - Universidad de Burgos - España

Jover Olmedo, Gonzalo - Universidad Complutense de Madrid - España

Kahn, Pierre - Université Caen Normandie - Francia

Lázaro Lorente, Luis Miguel - Universitat de València - España

Leñero Llaca, Marta - Universidad Autònoma Nacional de México - México

León Alvarez, Aaron - Universidad de La Laguna - España

Lluch Crespo, Gemma - Universitat de València - España

López Facal, Ramón - Universidade de Santiago de Compostela - España

Lorenzo Vicente, Juan Antonio - Univesidad Complutense de Madrid - España

Luzón Trujillo, Antonio - Universidad de Granada - España

Magalhães, Justino - Universidade de Lisboa - Portugal (3 evaluaciones)

Mahamud Angulo, Kira - Universidad Nacional de Educación a Distancia - España

Marqués Sureda, Salomó - Universitat de Girona - España

Moreno Martínez, Pedro Luis - Universidad de Murcia - España

Oropeza Sandoval, Luciano - Universidad de Guadalajara - México

Ortuzar González, Mónica - Universidade de Vigo - España

Parra, David - Universitat de València - España

Pedraza Gracia, Manuel José - Universidad de Zaragoza - España

Pérez Garzón, Sisinio - Universidad de Castilla - La Mancha - España

Pérez - Aldeguer, Santiago - Universidad de Zaragoza - España

Pintassilgo, Joaquim - Universidade de Lisboa - Portugal

Polenghi, Simonetta - Università Cattolica del Sacro Cuore - Italia

Pozo Andrés, María del Mar - Universidad de Alcalá - España

Pozuelo Yvancos, José M. - Universidad de Murcia - España

Pujol Mongoy, Maite - Universitat de Vic - Universitat Central de Catalunya - España

(2 evaluaciones)

Raftery, Deirdre - University College Dublin - Irlanda 
Ramírez Hernández, Georgina - Universidad Nacional Autónoma de México - México Rogers, Rebecca - Université Paris Descartes - Francia

Saez Carreras, Juan - Universidad de Murcia - España

Sani, Roberto - Università di Macerata - Italia

Sevilla Merino, Diego - Universidad de Granada - España

Sierra Blas, Verónica - Universidad de Alcalá - España

Simon, Frank - Universiteit Gent - Bélgica (2 evaluaciones)

Soler Mata, Joan - Universitat de Vic - Universitat Central de Catalunya - España

Somoza, Miguel - Universidad Nacional de Educación a Distancia - España (2 evaluaciones)

Sureda García, Bernat - Universitat de les Illes Balears - España

Tambara, Elomar - Universidade Federal de Pelotas - Brasil

Terrón Bañuelos, Aida - Universidad de Oviedo - España

Tiana Ferrer, Alejandro - Universidad Nacional de Educación a Distancia - España Tröhler, Daniel - Université du Luxembourg - Luxemburgo

Valls, Rafael - Universitat de València - España

Vasconcelos, María Celi Chaves - Universidade do Estado do Rio de Janeiro - Brasil Vazquez Ramil, Raquel - Universidade de Vigo - España

Vergara Ciorda, Fernando Javier - Universidad Nacional de Educación a Distancia España

Vidal, Diana - Universidade de São Paulo - Brasil

Vilafranca Manguán, Isabel - Universitat de Barcelona - España

Vilanou Torrano, Conrad - Universitat de Barcelona - España

Viñao Frago, Antonio - Universidad de Murcia - España (2 evaluaciones) 